Article

\title{
A Survey of Neotropiella Handschin, 1942 (Collembola, Neanuridae, Pseudachorutinae) with the Description of a New Brazilian Species
}

\author{
Bruno C. Bellini ${ }^{1, *(\mathbb{D})}$, Wanda M. Weiner ${ }^{2} \mathbb{( D}$, Gabriel C. Queiroz ${ }^{3} \mathbb{D}$ and Raiane V. Paz ${ }^{1, *}$ \\ 1 Laboratório de Collembola, Departamento de Botânica e Zoologia, Centro de Biociências, Universidade Federal \\ do Rio Grande do Norte-UFRN. BR 101, Lagoa Nova, Campus Universitário, Natal 59072-970, Brazil \\ 2 Institute of Systematics and Evolution of Animals, Polish Academy of Sciences, Sławkowska 17, \\ Pl-31-016 Kraków, Poland; weiner@isez.pan.krakow.pl \\ 3 Museu Nacional, Departamento de Entomologia, Universidade Federal do Rio de Janeiro-UFRJ. Quinta da Boa \\ Vista, São Cristóvão, Rio de Janeiro 20940-040, Brazil; gabriel_cq@yahoo.com.br \\ * Correspondence: entobellini@gmail.com (B.C.B.); raianevital11@gmail.com (R.V.P.)
}

Received: 24 April 2020; Accepted: 3 June 2020; Published: 13 July 2020

check for updates

\begin{abstract}
Neotropiella Handschin, 1942 is a pantropical genus of Pseudachorutinae with 18 species, 16 of which are from the Neotropical Region and 13 from Brazil. The genus has several species with unclear descriptions. Herein, we describe a new species of Neotropiella, survey the genus based on published papers and discuss its morphology, providing an updated genus diagnosis plus a detailed comparison table and a key to all known species. Neotropiella arretada sp. nov. is unique in the combination of a postantennal organ with 14-20 vesicles, its mandible with five teeth, its maxilla apically pointed and its unguis with a pair of reduced teeth. Widely distributed taxa may be species complexes, especially due to their unclear descriptions.
\end{abstract}

Keywords: Aethiopella Handschin; chaetotaxy; identification key; Neanuroidea; plurichaetosis; review; taxonomy

\section{Introduction}

Neotropiella Handschin, 1942 was proposed as a subgenus of Aethiopella Handschin, 1942, to group species of the genus with five to seven eyes [1]. In 1949, Stach [2] raised Neotropiella to genus level, a position maintained by Massoud in his review of the Austral Neanuroidea [3]. After the transfer of N. malkini Arlé, 1981 to Sernatropiella Palacios-Vargas, 2019, it currently encompasses 18 pantropical species, of which 16 described were from the Neotropical Region [4-8]. The genus is one of the most representative and widespread groups of Pseudachorutinae (Neanuridae) in the Neotropics [6,9]. In Brazil, Neotropiella is the most diverse genus of Poduromorpha, with 13 recorded species: Neotropiella arlei Najt, Thibaud and Weiner, 1990; N. barbatae Queiroz, Silveira and Mendonça, 2013; N. carli (Denis, 1924); N. denisi (Arlé, 1939); N. digitomucronata Thibaud and Massoud, 1983; N. insularis Queiroz, Silveira and Mendonça, 2013; N. macunaimae Queiroz, Silveira and Mendonça, 2013; N. meridionalis (Arlé, 1939); N. minima Thibaud and Oliveira, 2010; N. plurichaetosa Thibaud and Oliveira, 2010; N. quinqueoculata (Denis, 1931); N. silvestrii (Denis, 1929); and N. vanderdrifti Massoud, 1963 [6,9-18]. Most Brazilian taxa have been recorded from humid forests or their surroundings within the Atlantic and Amazon forests domains $[6,9,12,14,18]$. 
Previous diagnoses of Neotropiella separate the genus from other Pseudachorutinae by the combination of the apical bulb of the fourth antennal segment trilobed, the sense rods of the third antennal segment organ within a single integumental cavity, the postantennal organ moruliform (with at least two rings of vesicles), $5+5$ or $6+6$ eyes [6], the maxilla styliform without fringed or toothed lamellae, and the furca present with developed dens and mucro $[3,6]$. Species with complex mandibles (with about eleven to twelve teeth) are the only ones to have $6+6$ eyes, which raises questions about their identities as Neotropiella taxa [6]. Nevertheless, the genus is similar to Arlesia Handschin, 1942 in the reduction of eyes, but the latter lacks postantennal organ [1,3]. Also the species with $5+5$ eyes of Neotropiella have large eyes, quite near to each other, while they are smaller and spaced further apart in Arlesia [3,6], and the features concerning antennal, body and leg chaetotaxy are dissimilar [7]. Neotropiella also resembles Aethiopella and Pseudachorutes Tullberg, 1871, but differs from both especially in having reduced eyes $(8+8$ in both genera) and a moruliform postantennal organ (a simple ring of vesicles on Pseudachorutes) $[3,19,20]$. The antennal and body chaetotaxy of Neotropiella is similar to that of Pseudachorutes, as it possesses a microsensillum (ms) on Ant. IV, little or no plurichaetosis on head and body, and $\mathbf{M}$ chaeta between $\mathbf{A}$ and $\mathbf{B}$ rows on tibiotarsus. These character states separate Neotropiella from the Neotropical genera with derived characteristics, such as the absence of $\mathbf{m s}$ on Ant. IV, paurochaetotic or plurichaetotic head and body (Arlesia and Handschinurida Queiroz, 2015 groups, respectively) and basally displaced M chaeta on tibiotarsus $[7,21]$.

Herein, we describe a new species of Neotropiella from Brazil, update the diagnosis of the genus, provide additions and corrections to the comparative table shown in [6], discuss the genus and provide a key to all known species.

\section{Materials and Methods}

The specimens were collected using pitfall traps during 2018, from forested areas within the Atlantic Forest phytogeographic domain in the Nísia Floresta municipality, Rio Grande do Norte state, Brazil. They were sorted under a stereomicroscope, preserved in $70 \%$ ethanol and stored at $6{ }^{\circ} \mathrm{C}$; posteriorly they were diaphanized in Nesbitt's solution at $50{ }^{\circ} \mathrm{C}$, washed in Arlés liquid and mounted on glass slides in Hoyer's solution [22,23]. Morphology analyses and line drawings were made with drawing tube attached to Leica DM500 and DM750 microscopes (Wetzlar, Germany). Photographs were taken with a Leica MC170HD camera (Wetzlar, Germany) attached to a DM750 microscope using LAS 4.12 software. Final figures were made on CorelDraw X8 software (23 April 2020). The type series is deposited at the Collembola Collection of Centro de Biociências of Universidade Federal do Rio Grande do Norte, Brazil (CC/UFRN), and at the Institute of Systematics and Evolution of Animals, Polish Academy of Sciences, Kraków, Poland (ISEA).

The terminology used to label structures follows: Yosii (1960) with additions of Jordana et al. (1997) to dorsal head and trunk chaetotaxy, with a few adaptations $[23,24]$. Dorsal thorax I chaetotaxy was only numbered due to its unclear homology as pointed out by Cassagnau [25]. Dorsal thorax II to abdomen IV fields of chaetae are labeled as proposed by Deharveng (1983) and used by Potapov and Banasco (1985), but with merging the dorsolateral (Dl) and lateral (L) fields on a single DL field [26,27]. Ventral abdomen II-V fields of chaetae follow Deharveng (1983) [26]. Labial chaetotaxy follows [3,26,28]. Dorsal sensilla on the fourth and third antennal segments and tibiotarsal chaetotaxy follows $[7,26,28]$.

Abbreviations used in the descriptions are as follows: Abd. = abdominal segment(s); Ant. = antennal segment(s); PAO = postantennal organ; Th. = thoracic segment(s). Chaetae labels are marked in bold. 


\section{Results}

\subsection{Taxonomic Summary and Genus Diagnosis}

Order Poduromorpha Börner, 1913 [29]

Superfamily Neanuroidea Massoud, 1967 sensu Deharveng, 2004 [3,30]

Family Neanuridae Börner, 1901 [31]

Subfamily Pseudachorutinae Börner, 1906 [32]

Genus Neotropiella Handschin, 1942 [1]

Diagnosis. Head and body pigmented, mostly dark blue or violet to black; eyepatches black; oral cone, legs and furca generally white to yellowish; distal antenna variably pigmented; body oval, compressed dorso-ventrally; paratergites and paratergal areas variably developed; Ant. III and IV strongly fused, ventral limit seen only in some species; Ant. IV variable in length, shorter, subequal to or longer than Ant. III; Ant. IV mostly with trilobed apical bulb, rarely four-lobed, dorsally with five to seven normal sensilla, microsensillum (ms) mostly present, ventrally with or without sensorial field; Ant. III organ composed of two short sensory rods protected by a well-developed cuticular fold, dorsal and ventral guard sensilla (Sgd and Sgv, respectively) present and subequal, ventral microsensillum (ms) present; oral cone elongated; mandible with three to six teeth; maxilla capitulum styliform formed by one to three lamellae entirely fused or divided apically (hooked); PAO moruliform, with at least two rings composed of 7-80 vesicles; $5+5$ large eyes, close to each other; Th. I with $2+2$ to $5+5$ dorsal chaetae, except for N. murphyi Massoud, 1964, which has $6+6$ central chaetae plus several lateral chaetae (plurichaetosis); unguis with one internal tooth, with or without one to two pairs of lateral teeth; unguiculus absent; ventral tube mostly with four chaetae on each side, rarely with three; tenaculum with three teeth on each ramus; furca present and complete, with well-developed dens and mucro, each dens with six chaetae, rarely with five or seven chaetae; anal spines absent (adapted and updated from [3,6,33,34]).

Type species. Neotropiella silvestrii (Denis, 1929) [16].

Remarks. Here we dismiss N. denisi and N. mirabilis (Handschin, 1929) [33] from Neotropiella due to their remarkably complex mandibles, with about 12 teeth and $6+6$ small eyes, a very different morphology compared to other Neotropiella species as previously noted by [6]. Both species should be considered as incertae sedis since they cannot be clearly placed in any other genus of Pseudachorutinae for now.

\subsection{Neotropiella arretada sp. nov. Paz, Bellini, and Queiroz}

Figures 1-3.

Type material. Holotype: female on slide (CC/UFRN): Brazil, Rio Grande do Norte State, Nísia Floresta municipality, "Lagoa Redonda" Farm, 06² $02^{\prime} 47.5^{\prime \prime}$ S 3511'42.3" W, 49 m, 16-18.vi.2018, pitfall trap, Paz, R.V and Carvalho, M.N.A coll. Paratypes (CC/UFRN): two females, one male and four subadults (juveniles) on slides, same data as holotype. Paratype (ISEA): one female on slide, same data as holotype. Paratypes (CC/UFRN): one male and one subadult on slides, same data as holotype, except for sampling date: 13-15.iv.2018.

Diagnosis. Ant. IV with three to four lobes on apical bulb, with six (S1-S4, S7-S8) dorsal sub-cylindrical sensilla, microsensillum (ms) present, ventral sensorial field absent, Ant. IV ventrally with nine broad, blunt chaetae; Ant. III-IV ventral separation marked; Ant. II and I with 11-12 and 7 chaetae, respectively; PAO with 14-20 vesicles; $5+5$ large eyes; mandible with five teeth; maxilla styliform with two lamellae completely fused, apically pointed; dorsal chaetotaxy mostly composed of primary chaetae (lacking clear plurichaetosis), at least some of dorsal chaetae barbed. Th. I with $4+4$ dorsal chaetae; tibiotarsus I-III with 19,19 and 18 chaetae, respectively; ungues with a pair of reduced lateral teeth; ventral tube with $4+4$ 
chaetae; manubrium with 10-12 chaetae; dens with six to seven dorsal chaetae; males with $4+4$ modified chaetae on the genital plate.
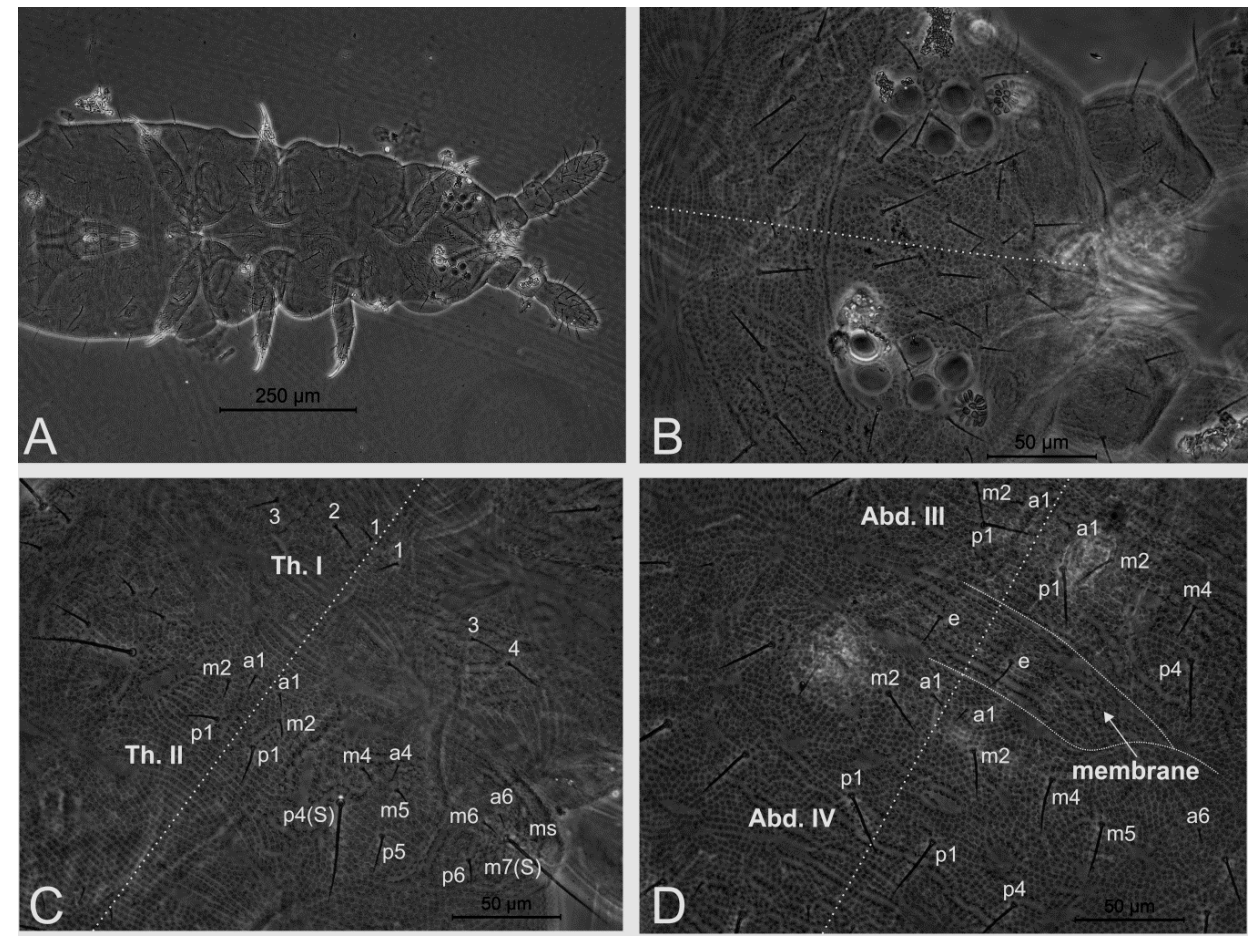

Figure 1. Neotropiella arretada sp. nov. holotype: (A) partial habitus (Abd. V and VI omitted); (B) dorsal head; (C) Th. I-II dorsal chaetotaxy (m2 absent on right side of Th. I, S = long sensilla); (D) Abd. III-IV partial dorsal chaetotaxy plus intersegmental membrane $(\mathrm{e}=$ extraordinary chaetae). Midline drawn over (B-D).

Description. Color in ethanol dark blue with black eyepatches; distal antennae, oral cone, legs and furca whitish. Habitus oval to elongated. Paratergites reduced (habitus "pseudochorutinian" sensu Massoud) [3] (Figure 1A). Body granules medium sized, uniformly distributed on tegument (Figure 1B-D). Body length of holotype: $1.15 \mathrm{~mm}$, range of type series (adults only) $0.88-1.53 \mathrm{~mm}$, females averaging $1.19 \mathrm{~mm}$, males $0.88 \mathrm{~mm}$, adults $1.09 \mathrm{~mm}$.

Head (Figures 1B and 2). Ratio cephalic diagonal: antenna in holotype $=1: 1.05$, in adult paratypes $=1: 1-1.08$. Ant. IV with trilobed apical bulb in most specimens, two paratypes with four-lobed apical bulb on only one antenna; dorsally with six subcylindrical sensilla (S1-S4, S7-S8), reduced i chaeta, dorsolateral microsensillum (ms) and subapical organite (or) present; ventrally without sensorial field but with nine broad, blunt chaetae (Figure 2A-C). Ant. III and IV dorsally fused, ventral separation marked (Figure 2C). Sensory organ of Ant. III with two small club-shaped sensilla, bent towards each other and protected by cuticular fold, surrounded by two longer and subcylindrical subequal guard sensilla, one dorsal and another ventral (Sgd and Sgv, respectively), ventral microsensillum (ms) present (Figure 2C). Ant. I and II with 7 and 11-12 chaetae, respectively. Mandible with five teeth, one basal and one subapical larger plus three smaller teeth (Figure 2D). Maxilla capitulum styliform, formed by two entirely fused lamellae (lateral one very reduced), apically pointed (Figure 2E). Clypeal area with $6+6$ and labrum of $2+2$ chaetae (Figure 2F). Oral cone elongated. Labial palp chaetae a1-a2 (A and C) longer than others (Figure 2G). Cephalic groove with $2+2$ surrounding postlabial chaetae (Figure $2 \mathrm{G}$ ). Eyes $5+5$, enlarged, anterior eye subequal to or slightly larger than others, eyepatches with three interocular chaetae (oc1-oc3) 
(Figures 1B and 2H). PAO moruliform, subequal to or slightly smaller than anterior eye, with 14-20 vesicles (Figure $2 \mathrm{H}$ ). Dorsal head chaetotaxy (Figure $2 \mathrm{H}$ ) with pointed micro (average $12 \mu \mathrm{m}$ in holotype) and slightly clavate mesochaetae (average $25 \mu \mathrm{m}$ in holotype), $\mathbf{d} \mathbf{0}$ and $\mathbf{d}^{\prime} \mathbf{0}$ as unpaired chaetae. Dorsal head chaetae slightly barbed.

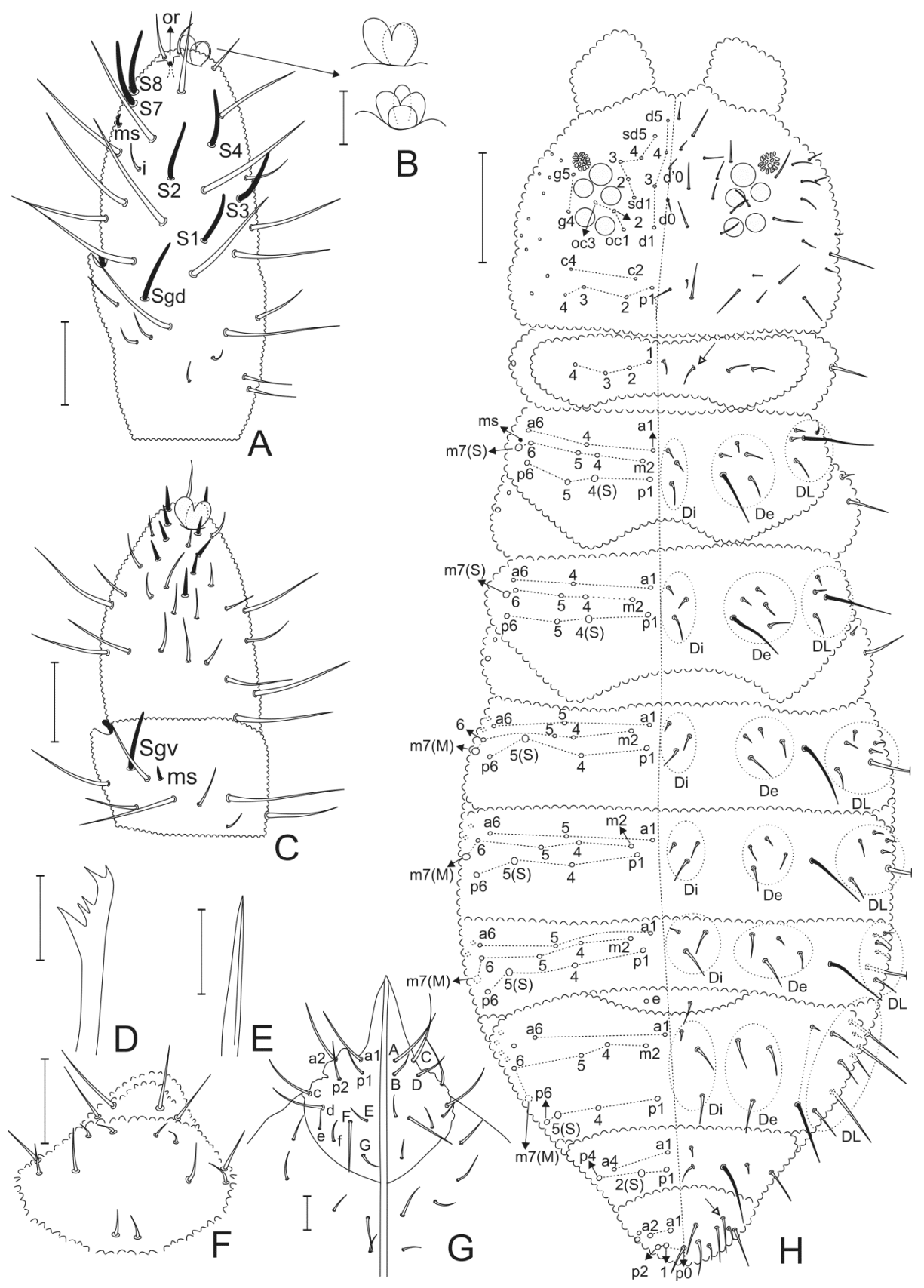

Figure 2. Neotropiella arretada sp. nov:: (A) left Ant. IV-III, dorsal view; (B) detail of different morphologies of Ant. IV apical bulb; (C) left Ant. III-IV, ventral view; (D) right mandible apex; (E) right maxilla capitulum; (F) clypeus and labrum morphology; (G) labial and postlabial chaetotaxy, left follows [28] and right [3] labial nomenclatures; $(\mathbf{H})$ body dorsal chaetotaxy, $\mathrm{S}=$ long sensilla, $\mathrm{M}=$ macrochaetae (white arrows point to chaetae present or absent). Scale bars: $5 \mu \mathrm{m}(\mathbf{D}, \mathbf{E}) ; 10 \mu \mathrm{m}(\mathbf{B}) ; 20 \mu \mathrm{m}(\mathbf{A}, \mathbf{C}, \mathbf{F}, \mathbf{G}) ; 100 \mu \mathrm{m}(\mathbf{H})$. 


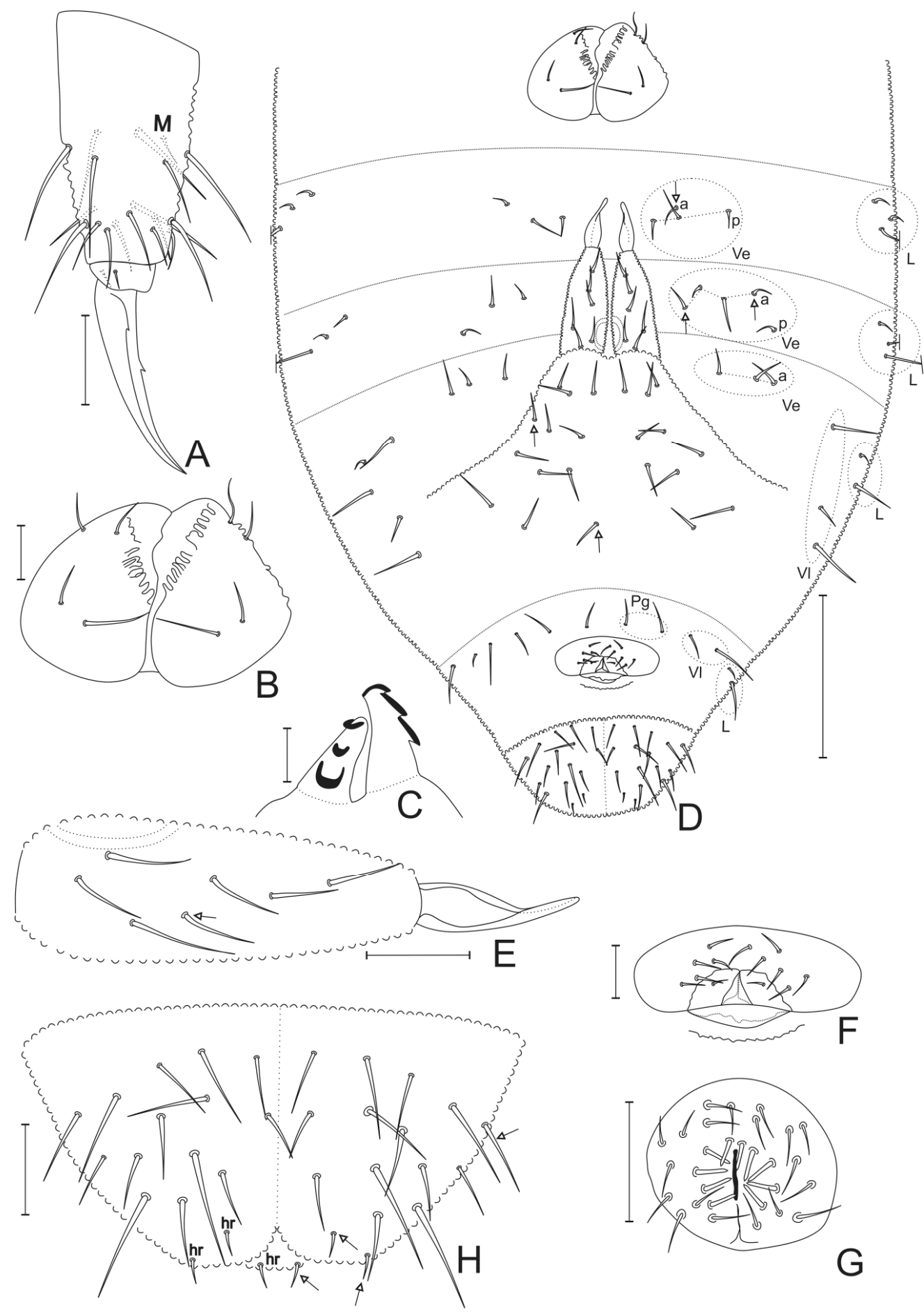

Figure 3. Neotropiella arretada sp. nov.: (A) tibiotarsus and empodial complex III (inner view); (B) ventral tube; (C) tenaculum rami and corpus; (D) ventral abdominal chaetotaxy, tenaculum omitted; (E) right dens and mucro, dorsal view; (F) female genital plate; (G) male genital plate; $(\mathbf{H})$ ventral anal valves and hr chaetae on dorsal anal valve. White arrows point to chaetae present or absent. Scale bars: $5 \mu \mathrm{m}(\mathbf{C})$; $10 \mu \mathrm{m}(\mathbf{F}) ; 20 \mu \mathrm{m}(\mathbf{A}, \mathbf{B}, \mathbf{E}, \mathbf{G}, \mathbf{H}) ; 100 \mu \mathrm{m}(\mathbf{D})$. 
Trunk dorsal chaetotaxy (Figures 1C,D and 2H). Dorsal thorax and abdomen with heterochaetosis formed by pointed microchaetae (average $8 \mu \mathrm{m}$ in holotype), slightly clavate mesochaetae (average $22 \mu \mathrm{m}$ in holotype), slightly clavate macrochaetae (average $37 \mu \mathrm{m}$ in holotype), and long sensilla (average $54 \mu \mathrm{m}$ in holotype) (Figures 1C,D and 2H). Most dorsal meso and macrochaetae slightly barbed. Dorsal trunk chaetotaxy mostly composed of primary chaetae (without plurichaetosis). Half trunk sensillar formula from Th. I to Abd. VI as 022/111110 (Figure 2H). Th. I with $4+4$ chaetae, holotype abnormal with $4+3, \mathbf{1}$ as microchaeta, $\mathbf{2}-\mathbf{4}$ as mesochaetae; Th. II to Abd. IV half trunk dorsointernal (Di)/dorsoexternal (De)/dorsolateral (DL) fields with Di 33/3333, De 55/4443 and DL 54/6668 chaetae, respectively (Figures $1 \mathrm{C}, \mathrm{D}$ and $2 \mathrm{H}$ ). Th. II-III chaetotaxy similar, with $3+3$ chaetae on a row (a1, a4 and a6), $5+5$ on $\mathbf{m}$ row $(\mathbf{m} 2, \mathbf{m} \mathbf{4}-\mathbf{m} 7)$ and $4+4$ on $\mathbf{p}$ row (p1, p4-p6); Th. II-III long sensilla as $\mathbf{p} 4$ and $\mathbf{m} 7$; lateral microsensillum (ms) present on Th. II (Figures $1 \mathrm{C}$ and $2 \mathrm{H}$ ). Abd. I-III main chaetotaxy similar, with $3+3$ chaetae on a row (a1, a4 and a6), $5+5$ on $\mathbf{m}$ row $(\mathbf{m} \mathbf{2}, \mathbf{m} 4-\mathbf{m} 7)$ and $4+4$ on $\mathbf{p}$ row (p1, p4-p6), long sensillum as $\mathbf{p} 5, \mathrm{~m} 7$ as macrochaeta. A pair of extra chaetae (e chaetae) on the membrane between Abd. III and IV (Figures 1D and 2H). Abd. IV main chaetotaxy with $2+2$ chaetae on a row (a1, a6), $5+5$ on $\mathbf{m}$ row $(\mathbf{m} 2, \mathbf{m} 4-\mathbf{m} 7)$ and $4+4$ on $\mathbf{p}$ row (p1, p4-p6), long sensillum as $\mathbf{p} 5, \mathbf{m} 7$ as macrochaeta, a5 absent. Abd. $V$ main chaetotaxy with $2+2$ on a row (a1 and $\mathbf{a} 4)$ and $3+3$ on $\mathbf{p}$ row (p1, p2 and $\mathbf{p} 4)$, long sensillum as p2. Abd. VI with unpaired mesochaeta p0 (Figure 2H). Pseudopores not seen, apparently missing.

Trunk appendages and ventral abdomen (Figure 3). Chaetotaxy of legs I-III: Subcoxa I, 1/2/2; subcoxa II, 0/2/2; coxa, 3/8/7; trochanter, 5/5/5; femur, 12/11/10; tibiotarsus, 19/19/18. Tibiotarsus M chaeta present, aligned to B row or slightly more distal (Figure 3A). Unguis with a single median inner tooth, laterally with a pair of reduced proximal teeth; anterior and posterior pretarsal chaetae present (Figure 3A). Ventral tube with $4+4$ chaetae (Figure 3B). Tenaculum with three teeth on each ramus (Figure 3C). Abdominal segments I-V ventrally with $0 / 3-4 / 3-5 / 3 / 4$ central chaetae (excluding genital plates) by half body, respectively. Abd. II-IV half body ventral (Ve)/ventrolateral (Vl)/lateral (L) fields with: Ve 3-4(0-1a $+3 p) / 3-5(2-4 a+1 p) / 3(3 a), V l$ 0/0/3, L 3/3/2 chaetae, respectively (Figure 3D). Furca well developed: manubrium with 10-12 chaetae on each side; each dens with six chaetae (one latero-proximal longer than others), except for two paratypes with seven chaetae; mucro tapering at apex, average ratio mucro: dens of holotype $=2.31$ (Figure 3D,E). Female genital plate with $1+1$ smaller eugenital chaetae plus about 11 larger circumgenital chaetae (Figure 3F). Abd. V ventrally with $2+2$ pregenital $(\mathrm{Pg}), 2+2$ ventrolateral $(\mathrm{Vl})$ and $2+2$ lateral (L) chaetae (Figure 3D); male genital plate with $5+5$ thick modified eugenital chaetae plus about 19 circumgenital chaetae (Figure 3G); Abd. V ventrally with $3+3$ pregenital, $2+2$ ventrolateral and $2+2$ lateral chaetae. Paired ventral anal valves with 13-14 ordinary chaetae plus two rear margin (hr) microchaetae each (holotype lacking both $\mathrm{hr}$ microchaetae on right anal valve) (Figure 3D,H); dorsal anal valve with 13-15 ordinary chaetae plus $1+1 \mathrm{hr}$ microchaetae (holotype lacking $1 \mathrm{hr}$ microchaeta on right side) (Figures $2 \mathrm{H}$ and $3 \mathrm{H}$ ).

Etymology. "Arretada" or "arretado" is a regional expression commonly used in northeastern Brazil which means "nice".

Distribution and Habitat. Specimens of Neotropiella arretada sp. nov. were collected from sand and litter samples near a freshwater lagoon next to a forested area, about $9 \mathrm{~km}$ from the seashore. The sampled area belongs to the Atlantic Forest phytogeographic domain. It is the same type of locality as the recently described Aethiopella ricardoi Paz, Queiroz and Bellini, 2019 [20].

Remarks. Neotropiella arretada sp. nov. closely resembles N. arlei, N. barbatae and N. minima in having Ant. IV with six dorsal sensilla plus ms, sensorial field absent on ventral side of Ant. IV, Ant I-II with 7 and 11-12 chaetae, respectively, dorsal trunk lacking plurichaetosis, plus tibiotarsi I-III with 19, 19 and 18 chaetae, respectively (unknown to N. minima). However, the new species differs from them by having 14-20 vesicles on the PAO (25 or more in N. arlei and N. barbatae, 7-10 in N. minima), five teeth on the mandible (four in N. arlei and N. minima, six in N. barbatae), the apex of the maxilla pointed (hooked in 
N. arlei and N. barbatae), and $4+4$ chaetae on the dorsal Th. I $(3+3$ in N. arlei and N. minima). Compared to N. barbatae, the new species also has one extra chaeta on the De fields from Th. II to Abd. III. Among the above cited species, Neotropiella arretada sp. nov. is possibly more closely related to N. minima, due to their shared reduction of PAO vesicles. These two species can also be separated by the shape of the ventral modified chaetae on Ant. IV (blunt in the new species vs. somewhat truncate in N. minima).

\subsection{Identification Key* and Distribution ${ }^{* *}$ of Neotropiella Handschin, 1942 Species}

1. Unguis with two pairs of lateral teeth ... 2

- Unguis with one pair or devoid of lateral teeth ... 4

2. PAO with 30 or less vesicles, maxilla apex hooked, unguis teeth normally developed ... N. gordae Diaz and Najt, 1995; Venezuela

- PAO with 50 or more vesicles, maxilla apex pointed, unguis teeth strongly developed ... 3

3. PAO with 50-65 vesicles, South America distribution ... N. carli (Denis, 1924)*; Brazil, French Guiana, Guyana, Peru, Venezuela

- PAO with 65-70 vesicles, Malaysia distribution ... N. murphyi Massoud, 1964***; Malaysia

4. Dorsal chaetotaxy of plurichaetotic type ... N. plurichaetosa Thibaud and Oliveira, 2010; Brazil

- Dorsal chaetotaxy of normal type (mostly composed of primary chaetae) ... 5

5. Ant. IV sensorial field present, with 40 or more modified chaetae ... 6

- Ant. IV sensorial field absent, Ant. IV with 20 or less ventral modified chaetae ... 9

6. Ant. IV sensorial field with more than 140 modified chaetae, PAO with $38-40$ vesicles ... N. insularis Queiroz, Silveira and Mendonça, 2013; Brazil

- Ant. IV sensorial field with about 76 or less modified chaetae, PAO with less than 34 or more than 50 vesicles ... 7

7. Ant. IV sensorial field with 40 modified chaetae, PAO with 50-60 vesicles, mandible with 5 teeth, maxilla apex hooked ... N. duranti Diaz and Najt, 1995; Venezuela

- Ant. IV sensorial field with 46 or more modified chaetae, PAO with 34 or less vesicles, mandible with 4 teeth, maxilla apex pointed ... 8

8. Ant. IV sensorial field with 76 modified chaetae, PAO with 34 vesicles, De field with 5 chaetae on Th. II and III ... N. digitomucronata Thibaud and Massoud, 1983; Brazil, Ecuador, Guadeloupe, Venezuela

- Ant. IV sensorial field with 46 modified chaetae, PAO with 20-22 vesicles, De field with 4 chaetae on Th. II and III ... N. pedisensilla Najt, Thibaud and Weiner, 1990; French Guiana

9. Th. I with $4+4$ chaetae ... 10

- Th. I with $3+3$ or $2+2$ chaetae ... 12

10. Ant. IV with 7 dorsal sensilla and 7-8 ventral modified chaetae ... N. macunaimae Queiroz, Silveira and Mendonça, 2013; Brazil

- Ant. IV with 6 dorsal sensilla and 9 or more ventral modified chaetae ... 11

11. Ant. IV with 18-20 ventral modified chaetae, PAO with 27-29 vesicles, mandible with 6 teeth, maxilla apex hooked, De field with 4 chaetae on Th. II and III ... N. barbatae Queiroz, Silveira and Mendonça, 2013; Brazil

- Ant. IV with 9 ventral modified chaetae, PAO with 14-20 vesicles, mandible with 5 teeth, maxilla apex pointed, De field with 5 chaetae on Th. II and III ... N. arretada sp. nov.; Brazil 
12. Adult specimens large sized (more than $3 \mathrm{~mm}$ ), Ant. IV with 9 ventral modified chaetae, PAO with 25-32 vesicles, De field with 4 chaetae on Th. II and III ... N. arlei Najt, Thibaud and Weiner, 1990; Brazil, French Guiana

- Adult specimens small to medium sized ( $2 \mathrm{~mm}$ or less), Ant. IV with 8 or less ventral modified chaetae, PAO with 23 or less vesicles, De field with 5 chaetae on Th. II and III ... 13

13. Ant. IV with 7 dorsal sensilla, PAO with 18-23 vesicles, mandible with 6 teeth, Th. I with $2+2$ chaetae, De field with 4 chaetae on Abd. I-III ... N. vanderdrifti Massoud, 1963; Neotropical

- Ant. IV with 6 dorsal sensilla, PAO with 7-10 vesicles, mandible with 4 teeth, Th. I with $3+3$ chaetae, De field with 2-3 chaetae on Abd. I-III ... N. minima Thibaud and Oliveira, 2010; Brazil

* Neotropiella carli, N. meridionalis (Brazil, Cuba), N. quinqueoculata (Neotropical) and N. silvestrii (Neotropical) have unclear descriptions, and are herein proposed as species inquirendae (see Section 4.1). While N. carli can be separated from most species due to the presence of two pairs of lateral teeth on its unguis, N. meridionalis, $N$. quinqueoculata and N. silvestrii are remarkably similar to other species in their few known features, so the last three species were excluded from the key

** widespread species distributed in six or more countries, including Central and South America, were considered with Neotropical distribution

*** see discussion topic 4.3

\section{Discussion}

\subsection{Species Inquirendae}

Neotropiella carli, N. meridionalis, N. quinqueoculata and N. silvestrii descriptions lack sufficient data to clearly diagnose each one (see Table 1 ). Such names should be used with caution, especially outside their type localities. Here we propose N. carli, N. meridionalis, N. quinqueoculata and N. silvestrii as species inquirendae, and these species must be urgently redescribed so their names can be confidently used. Neotropiella arlei's, $N$. digitomucronata's and $N$. vanderdrifti's morphologies are better understood, but they need to have more specimens from their type localities revised, since they were described based on only one or two type specimens (Table 1). For these three species at least, the range of the PAO number of vesicles should be used with caution, as the analysis of more specimens could extend it.

\subsection{Remarks on Neotropiella araguaensis Rapoport, and Maño, 1969}

Arlé listed the Brazilian species of Pseudachorutinae, and suggested that N. araguaensis from Venezuela could be a synonym of N. carli, based on their similar morphology and distribution (both are recorded from the Amazon Forest) [4]. He also suggested that N. carli was widely distributed, from northern Brazil and Peru, to Guyana and French Guyana, and so could possibly occur in Venezuela as well (the type locality of N. araguaensis) [4,35]. Diaz and Najt [36] officially proposed this synonym, apparently without further analysis or new data regarding N. araguaensis. The Mari Mutt and Bellinger's catalog of Neotropical springtails [37] provided Arlé's note, and in its supplements [38,39] endorsed his position, probably based on the mentioned synonymy [36]. The two species are similar, but several important features concerning their morphology and chaetotaxy are as yet unknown. Massoud [3] considered N. carli to have a trilobed apical bulb on Ant. IV and 65 vesicles in the PAO. The original description of $N$. araguaensis indicated a four-lobed apical bulb on Ant. IV, although in some specimens it appeared that one of the three main lobes is subdivided, and a PAO with 50-57 vesicles [35]. Since N. carli is species inquirenda (see Section 4.1) and $N$. araguaensis description lacks important data to diagnose it, we believe the available differences do not support the revalidation of $N$. araguaensis at this time, and it will remain as a synonym of $N$. carli. 
We therefore merged the morphology and distribution of both species under N. carli name in the key and in Table 1.

\subsection{Remarks on Neotropiella murphyi Massoud, 1964}

After our new diagnosis of Neotropiella, the sole species outside the Neotropical Region is N. murphyi, recorded only from Malaysia [8,34]. While this species shares important diagnostic features with other Neotropiella taxa, like $5+5$ large eyes and mandibles with a low number of teeth, among others, it has a unique type of plurichaetosis. Neotropiella murphy has a complex thoracic chaetotaxy, with several multiplets not seen even in N. plurichaetosa, the sole Neotropical species with confirmed plurichaetosis (see Table 1). Because of its distribution and dissimilar morphology, N. murphy may represent another genus arbitrarily included in Neotropiella. However, in the absence of further details, including its dorsal chaetotaxy which is insufficiently described by Massoud [34], we would rather keep this species as Neotropiella at this time.

\subsection{On the Widespread Species of Neotropiella}

At least three species of Neotropical Neotropiella are widely distributed in South and Central America: N. quinqueoculata, N. silvestrii and N. vanderdrifti, while N. carli is widely distributed in the Amazon and Atlantic Forest domains [9,37-39]. All four species were included in Massoud's key [3], but there is much information missing from these taxa (see Section 4.1 and Table 1). Neotropiella quinqueoculata is possibly the most widespread species of the four, and according to Arlé [4], it has a remarkably variable morphology, especially regarding its PAO. The name N. quinqueoculata may conceal a species complex which should be investigated. Because of this, we used Denis's drawings [15] to provide a reliable number of PAO vesicles for the species (Table 1). The names N. silvestrii, N. vanderdrifti and N. carli may also hide species complexes.

N. quinqueoculata cannot be clearly separated from N. silvestrii by most of its characters (see Table 1), except for habitus. Massoud pointed to the more "pseudachorutinian" habitus of N. quinqueoculata (with reduced paratergites), and to the more "ceratrimerian" habitus of N. silvestrii (with large paratergites) [3]. Although such differences can arguably be applied to separate the species, it is clear both need to be revised and redescribed, as stated in Section 4.1.

\subsection{Homology and Comparative Morphology of Neotropiella Species}

Neotropiella apparently shows a stable sensillar trunk chaetotaxy; all species with known dorsal chaetotaxy have a 022/111110 half tergum formula, as seen in N. arlei, N. barbatae, N. digitomucronata, N. insularis, N. macunaimae, N. minima, N. murphyi, N. pedisensilla Najt, Thibaud and Weiner, 1990, N. plurichaetosa, $N$. vanderdrifti and $N$. arretada sp. nov. $[6,10,13,14,17,34]$. Some of these species also possess extra chaetae on Abd. IV, just above a1. Massoud's drawing of $N$. vanderdrifti suggests these extra chaetae are actually on the intersegmental membrane, between Abd. III and Abd. IV ([17] p. 48, Figure 1D), exactly as seen in $N$. arretada sp. nov. (Figures $1 \mathrm{D}$ and $2 \mathrm{H}$ ). The species with known dorsal chaetotaxy mostly have one chaeta on each side, above a1, but $N$. arlei, N. digitomucronata and N. murphyi apparently lack such extra chaetae, and at least N. plurichaetosa shows two pairs of them ([14] p. 138, Figure 2C). Most species of Neotropiella have only primary chaetotaxy, but at least two of them show plurichaetosis: N. murphyi and N. plurichaetosa. However, such species differ in their general morphology (as shown in Table 1) and in the nature of their plurichaetosis, as discussed in the Section 4.3. Another possible plurichaetotic species is N. carli, which has never had its chaetotaxy properly studied, possibly due to the large size of adult specimens (usually larger than $5 \mathrm{~mm}$ ). However, it has a high density of chaetae on the antennae, including a well-developed sensorial field on Ant. IV, which may correlate with body plurichaetosis as well [40]. 
The large eyes of Neotropiella species are not clearly homologous compared to the Poduromorpha species with $8+8$ eyes. The two posterior eyes are quite possibly F-G, since they are below the intraocular row oc1-oc3 (although the proximity of the F eye to the lateral g4 chaeta suggests it can also be E), while designations for the three anterior eyes are unclear. Due to their proximity to the interocular chaetae, they could be eyes $\mathrm{C}-\mathrm{D}$ and $\mathrm{H}$, but due to their proximity to the PAO and sd2-3 chaetae, they could also be interpreted as eyes A-C. The two species with $6+6$ eyes, previously assigned as Neotropiella, N. denisi and N. mirabilis, do not share such enlarged eyes, and their homology is clearer: N. denisi has eyes A-C and E-G, and while Handschin's drawing of N. mirabilis's eyes is incomplete, it shows at least the eyes are clearly not enlarged ([33] p. 17, Figure 1D).

While several species of Neotropiella do not have a sensorial field on Ant. IV, most (if not all) do have modified ventral chaetae (see Table 1), which may be homologous to the sensorial field since they arise in the same antennal region. The species of Neotropiella with few modified ventral chaetae on Ant. IV are: N. arlei, N. barbatae, N. macunaimae, N. minima, N. plurichaetosa, N. vanderdrifti and N. arretada sp. nov. $[6,10,14,17]$. In the new species, such chaetae are more similar to the ones of N. arlei (broad and somehow pointed), and resemble some species in other Neanuroidea genera, such as Aethiopella, Arlesia, Pseudachorutella Stach, 1949 and Brachystomella Ågren, 1903 [2,3,20,41,42]. Even so, within Neotropiella, the presence or absence of a sensorial field cannot clearly delimit ingroups, since it does not appear to be congruent with other diagnostic features (Table 1).

\subsection{Notes on Biogeography of Neotropiella}

Most species of Neotropiella (if not all; see Section 4.3) are from the Neotropical Region, and all Neotropical taxa are found in South America [8,9,37-39]. This pattern strongly suggests South America is the center of origin of Neotropiella, and therefore the Andean Cordillera may have represented an important biogeographic barrier to the genus speciation and distribution in the Americas. The northern Andes barrier is mostly represented by Paramo province (high cordilleras, above $3000 \mathrm{~m}$ ) sensu Morrone [43], and its uplift started during the late Cretaceous (from about 100-66 mya) [44], but it became conspicuous more recently during the Miocene (23-7 mya) [45]. In this sense, Neotropiella may have arisen by at least 7 mya in the forested areas of South America, and its distribution in the Antilles and Central America could have occurred via the Caribbean Sea, similarly to the model proposed by Christiansen and Bellinger (1994) for the Hawaiin colonization of springtails [46]. To support this hypothesis, all species recorded in Mexico and the Antilles are also recorded in South America.

On the other hand, Neotropiella murphyi's dissimilar distribution could possibly be better explained by two hypotheses: (1) It is a Neotropical species not yet recorded in the Neotropical Region, seen in Malaysia due to recent human-mediated dispersal events like soil and plant transportation during the past five centuries. (2) It is not related to the Neotropical taxa, as suggested in Section 4.3. 
Table 1. Main features of Neotropiella Handschin, 1942 species.

\begin{tabular}{|c|c|c|c|c|c|c|c|c|c|c|c|c|c|c|c|c|c|c|c|}
\hline Species & $\begin{array}{c}\text { Number } \\
\text { of Type } \\
\text { Series } \\
\text { Specimens }\end{array}$ & $\begin{array}{c}\text { Size of Type } \\
\text { Series } \\
\text { Specimens in } \\
\text { mm }\end{array}$ & $\begin{array}{l}\text { Ant. IV } \\
\text { Sensilla }\end{array}$ & $\begin{array}{l}\text { Ant. IV } \\
\text { Sensorial } \\
\text { Field }\end{array}$ & $\begin{array}{l}\text { Ant. IV } \\
\text { Ventral } \\
\text { Modified } \\
\text { Chaetae } \\
\end{array}$ & $\begin{array}{c}\text { Ant. I } \\
\text { Chaetae }\end{array}$ & $\begin{array}{l}\text { Ant. II } \\
\text { Chaetae }\end{array}$ & $\begin{array}{c}\text { PAO } \\
\text { Vesicles }\end{array}$ & $\begin{array}{l}\text { Mandible } \\
\text { Teeth }\end{array}$ & $\begin{array}{c}\text { Maxilla } \\
\text { Apex }\end{array}$ & $\begin{array}{l}\text { Trunk } \\
\text { Chaetae }\end{array}$ & $\begin{array}{l}\text { Trunk } \\
\text { Plurichaetosis } * *\end{array}$ & $\begin{array}{c}\text { Th. I } \\
\text { Chaetae } \\
\text { (per Side) }\end{array}$ & $\begin{array}{c}\text { Di Chaetae on } \\
\text { Th. II-III/Abd. } \\
\text { I-III }\end{array}$ & $\begin{array}{l}\text { De Chaetae } \\
\text { on Th. } \\
\text { II-III/Abd. } \\
\text { I-III }\end{array}$ & $\begin{array}{l}\text { Extra Chaetae } \\
\text { (e) between } \\
\text { Abd. III-IV } \\
\text { (per Side) }\end{array}$ & $\begin{array}{c}\text { Tibiotarsus } \\
\text { I-III } \\
\text { Formula }\end{array}$ & $\begin{array}{c}\text { Unguis } \\
\text { Lateral } \\
\text { Teeth }\end{array}$ & $\begin{array}{l}\text { Manubrium } \\
\text { Chaetae }\end{array}$ \\
\hline arlei $[6,10]$ & 2 & 3.4 & 6 & - & 9 & 7 & 11 & $25-32$ & 4 & $\mathrm{~h}$ & s? & - & 3 & $3 / 3$ & $4 / 3$ & $0 ?$ & $19 / 19 / 18$ & $+(2)$ & $?$ \\
\hline barbatae [6] & 3 & $0.8-1.15$ & 6 & - & $\begin{array}{l}18-20 \\
\end{array}$ & 7 & 12 & $27-29$ & 6 & $\mathrm{~h}$ & $\mathrm{~b}$ & - & 4 & $3 / 3$ & $4 / 3$ & 1 & 19/19/18 & - & 9 \\
\hline $\begin{array}{l}\text { carli } \\
{[3,6,11,35]}\end{array}$ & 1 & $4.3-6.0^{* * *}$ & $?$ & + & over 100 & $?$ & $?$ & $50-65$ & $3-4$ & $\mathrm{p}$ & s? & $+?$ & $?$ & $?$ & $?$ & $?$ & 18/18/17? & $+(4)$ & $?$ \\
\hline $\begin{array}{l}\text { digitomucronata } \\
{[6,13]}\end{array}$ & 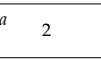 & 0.7 & $?$ & + & 76 & $?$ & $?$ & 34 & 4 & $\mathrm{p}$ & $\mathrm{s} ?$ & - & 5 & $3 / 3$ & $5 / 2(1)$ & 1 & $?$ & $+(2)$ & $?$ \\
\hline $\begin{array}{l}\text { duranti } \\
{[6,36]}\end{array}$ & 6 & $2.19-2.59$ & 7 & + & 40 & 7 & 11 & $50-60$ & 5 & $\mathrm{~h}$ & s? & - & $?$ & ? & $?$ & $0 ?$ & 18/18/17 & $+(2)$ & $?$ \\
\hline $\begin{array}{l}\text { gordae } \\
{[6,36]}\end{array}$ & 12 & $2.4-3.57$ & 5 & + & $28-35$ & 10 & 13 & $26-30$ & 4 & $\mathrm{~h}^{*}$ & s? & - & $?$ & $?$ & $?$ & $?$ & $18 / 18 / 17$ & $+(4)$ & $?$ \\
\hline insularis [6] & 20 & $2.5-3.8$ & 6 & + & over 140 & 9 & 12 & 38-40 & 5 & $\mathrm{~h}$ & $\mathrm{~s}$ & - & 4 & $3 / 3$ & $4 / 3$ & 1 & $19 / 19 / 18$ & - & 15-16 \\
\hline $\begin{array}{l}\text { macunaimae } \\
{[6]}\end{array}$ & 15 & $0.8-2.23$ & 7 & - & $7-8$ & 7 & 12 & $23-27$ & 5 & $\mathrm{~h}$ & $\mathrm{~b}$ & - & 4 & $3 / 3$ & $4 / 3$ & 1 & 19/19/18 & - & 9-10 \\
\hline $\begin{array}{l}\text { meridionalis } \\
{[3,6,12]}\end{array}$ & 4 & $1.3-3.2$ & $?$ & $?$ & $?$ & $?$ & $?$ & 29-30 & 4 & $\mathrm{p}$ & $?$ & $?$ & $?$ & $?$ & $?$ & $?$ & $?$ & $+(2)$ & $?$ \\
\hline $\begin{array}{l}\text { minima } \\
{[6,14]} \\
\end{array}$ & 11 & $0.35-0.65$ & 6 & - & $6-8$ & 7 & $11-12$ & $7-10$ & 4 & $\mathrm{p}$ & s? & - & 3 & $3 / 4$ & $5 / 3(2)$ & $1 ?$ & $?$ & $+(2)$ & $?$ \\
\hline $\begin{array}{l}\text { murphyi } \\
{[3,6,34]}\end{array}$ & 10 & up to 6.0 & $?$ & + & $?$ & ? & ? & $65-70$ & $4-5$ & $\mathrm{p}$ & s? & + & $6 \mathrm{~m}$ & $3 / 3$ & $9 / 7(5)$ & 0 & $18 / 18 / 17$ & $+(4)$ & $?$ \\
\hline $\begin{array}{l}\text { pedisensilla } \\
{[[6,10]}\end{array}$ & 3 & 1.1 & 6 & + & 46 & 7 & 12 & $20-22$ & 4 & $\mathrm{p}$ & s? & - & 4 & $3 / 3$ & $4 / 3$ & $0 ?$ & 19/19/18 & $+(2)$ & $?$ \\
\hline $\begin{array}{l}\text { plurichaetosa } \\
{[6,14]}\end{array}$ & 7 & $0.6-1.2$ & 6 & - & $5-8$ & 7 & $11-12$ & $27-36$ & $5-6$ & $\mathrm{p}$ & s? & + & 4 & $5(6) / 6$ & $6(7) / 7$ & 2 & $?$ & $+(2)$ & $?$ \\
\hline $\begin{array}{l}\text { quinqueoculata } \\
{[3,6,15]}\end{array}$ & 3 & up to 1.3 & $?$ & $?$ & $?$ & $?$ & $?$ & 33-54 & 4 & $\mathrm{p}$ & s? & $?$ & $?$ & $?$ & $?$ & $?$ & $?$ & $+(2)$ & $?$ \\
\hline $\begin{array}{l}\text { silvestrii } \\
{[3,6,16,47]}\end{array}$ & 5 & $2.5-3.0$ & $?$ & + & 29 & $?$ & $?$ & 35-50 & 4 & $\mathrm{p}$ & $?$ & $?$ & $?$ & $?$ & $?$ & $?$ & $?$ & $+(2)$ & $?$ \\
\hline $\begin{array}{l}\text { vanderdriffi } \\
{[3,6,17]}\end{array}$ & 1 & 2.0 & 7 & - & 6 & $?$ & $?$ & $18-23$ & 6 & p? & s? & - & 2 & $2(3) / 3(2)$ & $5 / 4$ & 1 & 18/18/17? & $+(2)$ & $?$ \\
\hline $\begin{array}{l}\text { arretada sp. } \\
\text { nov. }\end{array}$ & 11 & $0.88-1.53$ & 6 & - & 9 & 7 & ${ }^{11-12}$ & $14-20$ & 5 & $\mathrm{p}$ & $\mathrm{b}$ & - & 4 & $3 / 3$ & $5 / 4$ & 1 & 19/19/18 & $+(2)$ & 10-12 \\
\hline
\end{tabular}

Legends: [] species references; (+) present; (-) absent; (?) unknown/doubtful; (p) pointed; (h) hooked; (s) smooth; b (barbed); (m) plus several lateral multiplets; $\left({ }^{*}\right)$ trilamelate; $\left.{ }^{* *}\right)$ plurichaetosis of N. murphyi and N. plurichaetosa is apparently not homologous (see Section 4.3$) ;(* * *)$ size of type series specimens of N. araguaensis were also included, see the discussion. 


\section{Conclusions}

Neotropiella is a pantropical genus of Pseudachorutinae, with most of its known species being from the Neotropical Region. Several taxa lack properly detailed diagnoses, which compromise the differentiation of some of the species. We gathered further data from the original descriptions and revisions to better delimit them, but at least $N$. carli, N. meridionalis, N. quinqueoculata and N. silvestrii were herein considered species inquirendae. The new diagnosis of Neotropiella dismisses the species with $6+6$ eyes and complex mandibles, $N$. denisi and $N$. mirabilis, both of which are in need of redescription. The detailed description of $N$. arretada sp. nov. may be used as a model for future descriptions or revisions of the Neotropiella species. Widespread taxa, like N. quinqueoculata, N. silvestrii, N. vanderdrifti and N. carli may consist of several cryptic species. Following our research there are now 17 species of Neotropiella, 13 of them from Brazil.

Author Contributions: Conceptualization, B.C.B. and R.V.P.; methodology, R.V.P. and B.C.B.; software, R.V.P. and B.C.B.; validation, B.C.B., R.V.P., W.M.W. and G.C.Q.; formal analysis, R.V.P. and B.C.B.; investigation, R.V.P. and B.C.B.; resources, B.C.B.; data curation, B.C.B. and R.V.P.; writing-original draft preparation, B.C.B. and R.V.P.; writing-review and editing, B.C.B., W.M.W. and G.C.Q.; visualization, B.C.B., R.V.P., W.M.W. and G.C.Q.; supervision, B.C.B., W.M.W. and G.C.Q.; project administration, B.C.B.; funding acquisition, B.C.B. All authors have read and agreed to the published version of the manuscript.

Funding: This research was supported by UFRN. B.C.B. was granted by CAPES and CNPq (PROTAX II, process \#156828/2016-01 and PQ2018, process \# 305426/2018-4, respectively); G.C.Q. was granted by CAPES (PNDP scholarship) and CNPq (PDJ scholarship \#150451/2015-6 and AVG grant \#451149/2016-5); R.V.P. was granted by PIBIC/CNPq scholarship.

Acknowledgments: We thank Maria Neide Araújo de Carvalho for helping with the collections and the anonymous referees for the revision of this manuscript.

Conflicts of Interest: The authors declare no conflict of interest.

\section{References}

1. Handschin, E. Materialen zur Revision de Collembolen. Die Gattung Ceratrimeria C.B. sensu Womersley. Ver. Nat. 1942, 53, 265-284.

2. Stach, J. The Apterygotan Fauna of Poland in Relation to the World-Fauna of This Grup of Insects. Families: Anuridae and Pseudachorutidae, 1st ed.; Polska Akademia Umiejetnosci, Acta Monographica Musei Historiae Naturalis: Kraków, Poland, 1949; pp. 1-122.

3. Massoud, Z. Monographie des Neanuridae, Collemboles Poduromorphes apiéces buccales modifiées. In Biologie de l'Amerique Australe, 1st ed.; Delamare Deboutteville, C., Rapoport, E.H., Eds.; Éditions du CNRS: Paris, France, 1967; Volume 3, pp. 7-399.

4. Arlé, R. Conspecto das espécies brasileiras de Pseudachorutinae, com descrição de uma espécie nova da Colômbia (Insecta, Collembola). Acta Amaz. 1981, 11, 583-593. [CrossRef]

5. Palacios-Vargas, J.G. An extraordinary new genus and species of Pseudachorutinae (Collembola: Neanuridae) from Colombia. Zootaxa 2019, 4609, 373-387. [CrossRef] [PubMed]

6. Queiroz, G.C.; Silveira, T.C.; Mendonça, M.C. New species of Neotropiella Handschin, (1942) (Collembola: Neanuridae) from Brazil. Soil Org. 2013, 85, 41-49.

7. Queiroz, G.C.; Zeppelini, D. Neotropical Pseudachorutinae (Hexapoda: Collembola: Neanuridae): A comparative morphological study with emphasis on endemic taxa. Zool. Anz. 2017, 269, 127-154. [CrossRef]

8. Bellinger, P.F.; Christiansen, K.A.; Janssens, F. Checklist of the Collembola of the World. Available online: http://www.collembola.org (accessed on 20 March 2020).

9. Zeppelini, D.; Queiroz, G.C.; Bellini, B.C. Collembola in Catálogo Taxonômico da Fauna do Brasil. PNUD. Available online: http:/fauna.jbrj.gov.br/fauna/faunadobrasil/379/ (accessed on 20 March 2020).

10. Najt, J.; Thibaud, J.-M.; Weiner, W. Collemboles (Insecta) Poduromorphes de Guyane française. Bull. Mus. Natl. Hist. Nat. Paris 1990, 12, 95-121.

11. Denis, J.R. Sur les collemboles du Muséum de Paris (1 ${ }^{\text {re }}$ partie). Ann. Soc. Entomol. Fr. 1924, 93, $211-260$. 
12. Arlé, R. Novas espécies de Pseudachorutini (Collembola) do Rio de Janeiro e arredores. Bol. Biol. Nova Ser. 1939, 4, 67-72.

13. Thibaud, J.-M.; Massoud, Z. Les Collemboles des Petites Antilles. III.-Neanuridae (Pseudachorutinae). Rev. Ecol. Biol. Sol. 1983, 20, 111-129.

14. Thibaud, J.-M.; Oliveira, E.P. Note sur les Collemboles de L'Amazonie Brésilienne II -Neanuridae: Pseudachorutinae ad. p. avec la description de deux espèces nouvelles. Rev. Fr. d'Entomol. 2010, 32, 135-140.

15. Denis, J.R. Contributo alla conoscenza del "Microgenton" di Costa Rica. II -Collemboles de Costa Rica avec une contribution au species de l'ordre. Boll. Lab. Entomol. Agr. 1931, 25, 69-170.

16. Denis, J.R. Notes sur les Collemboles récoltés dans ses voyages par la Professeur F. Silvestri (Descriptions d'espèces nouvelles). I-Collemboles d'Extreme-Orient. Boll. Lab. Entomol. Agr. 1929, 22, 166-180.

17. Massoud, Z. Les collemboles poduromorphes du surinam. Bull. Mus. Natl. Hist. Nat. Paris 1963, $20,42-51$.

18. Abrantes, E.A.; Bellini, B.C.; Bernardo, A.N.; Fernandes, L.H.; Mendonça, M.C.; Oliveira, E.P.; Queiroz, G.C.; Sautter, K.D.; Silveira, T.C.; Zeppelini, D. Errata Corrigenda and update for the 'Synthesis of Brazilian Collembola: An update to the species list.' Abrantes et al., (2010). Zootaxa, 2388: 1-22. Zootaxa 2012, 3168, 1-21. [CrossRef]

19. Tullberg, T. Förteckning öfver Svenska Podurider. Öfvers. K. VetenskAkad. Förh. 1871, 28, $143-155$.

20. Paz, R.V.; Queiroz, G.C.; Bellini, B.C. A new species of Aethiopella Handschin, 1942 (Collembola, Poduromorpha, Neanuridae) from Neotropical Region, with comments on the genus. Zootaxa 2019, 4629, 39-50. [CrossRef] [PubMed]

21. Queiroz, G.C. Handschinurida nom. nov. (Collembola, Neanuridae), a substitute name for the homonym Handschinia Stach, 1949. Zootaxa 2015, 4021, 499-500. [CrossRef]

22. Arlé, R.; Mendonça, C. Estudo preliminar das espécies de Dicranocentrus Schött, 1893, ocorrentes no Parque Nacional da Tijuca, Rio de Janeiro (Collembola). Rev. Bras. Biol. 1982, 42, 41-49.

23. Jordana, R.; Arbea, J.I.; Simón, C.; Luciáñez, M.J. Fauna Iberica Vol. 8: Collembola, Poduromorpha, 1st ed.; Museo Nacional de Ciencias Naturales, Consejo Superior de Investigaciones Científicas: Madrid, Spain, 1997; pp. 1-807.

24. Yosii, R. Studies on the Collembolan genus Hypogastrura. Amer. Midland Nat. 1960, 64, 257-281. [CrossRef]

25. Cassagnau, P. Chétotaxie et phylogénie chez les Collemboles Poduromorphes. Pedobiologia 1974, 14, 300-312.

26. Deharveng, L. Morphologie évolutive des Collemboles Neanurinae en particulier de la lignée Néanurienne. Trav. Lab. Écobiol. Arthr. Édaph. Toulose 1983, 4, 1-63.

27. Potapov, M.B.; Banasco, J. A new species of springtails from Cuba with comments on the role of chaetotaxy in diagnostic of Friesea spp. (Collembola: Neanuridae) (in Russian). Zool. Zhurnal 1985, 64, 1162-1167.

28. D'Haese, C.A. Homology and morphology in Poduromorpha (Hexapoda, Collembola). Eur. J. Entomol. 2003, 101, 385-407. [CrossRef]

29. Börner, C. Die Familien der Collembolen. Zool. Anz. 1913, 7, 315-322.

30. Deharveng, L. Recent advances in Collembola systematics. Pedobiologia 2004, 48, 415-433. [CrossRef]

31. Börner, C. Apterygoten-Fauna von Bremen und der Nachbardistrikte. Abh. Nat. Verein Bremen 1901, 17, 1-142.

32. Börner, C. Das System der Collembolen-Nebst Beschreibung neuer Collembolen des Hamburger Naturhistorischen Museums. Jahr. Hamburg. Wissen. Anst. 1906, 23, 147-186.

33. Handschin, E. Collembola from Abyssinia. Trans. Entomol. Soc. London 1929, 77, 15-28. [CrossRef]

34. Massoud, Z. Um nouveau Collembole Poduromorpe de Malaisie. Rev. Ecol. Biol. Sol 1964, 4, 701-704.

35. Rapoport, E.H.; Maño, S. Colembolos de Venezuela. I. Acta Biol. Venez. 1969, 6, 117-128.

36. Díaz, A.; Najt, J. Collemboles (Insecta) des Andes vénézuéliennes. Bull. Mus. Natl. Hist. Nat. Paris 1995, 16, 417-435.

37. Mari Mutt, J.A.; Bellinger, P.F. A Catalog of Neotropical Collembola, Including Nearctic Areas of Mexico. Flora E Fauna Handbook 5, 1st ed.; Sandhill Crane Press: Gainesville, FL, USA, 1990; pp. 1-237.

38. Mari Mutt, J.A.; Bellinger, P.F. Supplement to the catalog of the Neotropical Collembola. Caribb. J. Sci. 1996, 32, 166-175. 
39. Mari Mutt, J.A.; Bellinger, P.F.; Janssens, F. Checklist of the Collembola: Supplement to the Catalog of the Neotropical Collembola. Available online: http://www.collembola.org/publicat/neotrcat.htm (accessed on 20 March 2020).

40. Arlé, R. Collemboles d'Amazonie. I-Poduromorphes nouveaux ou peu connus et notes biologiques sur Neotropiella carli (Denis). Bol. Mus. Paraense Emílio Goeldi Belém N. S. Zool. 1966, 60, 1-19.

41. Ågren, H. Diagnosen einiger neuen Achorutiden aus Schweden (Vorläufige Mittheilungen). Entomol. Tidskrift 1903, 24, 126-128.

42. Bellini, B.C.; Santos, N.M.C.; Souza, P.G.C.; Weiner, W.M. Two new species of Brazilian springtails (Hexapoda: Collembola) with comments on Neotropical Brachystomella Ågren and Seira (Lepidocyrtinus) Börner. Insect Syst. Evol. 2019, 50, 297-326. [CrossRef]

43. Morrone, J.J. Biogeographical regionalisation of the Neotropical region. Zootaxa 2014, 3782, 1-110. [CrossRef] [PubMed]

44. Mescua, J.; Giambiagi, L.; Ramos, V. Late Cretaceous Uplift in the Malargüe fold-and-thrust belt (35'5), southern Central Andes of Argentina and Chile. Andean Geol. 2013, 40, 102-116. [CrossRef]

45. Morrone, J.J. Cladistic biogeography of the Neotropical region: Identifying the main events in the diversification of the terrestrial biota. Cladistics 2014, 30, 202-214. [CrossRef]

46. Christiansen, K.A.; Bellinger, P. Biogeography of Hawaiian Collembola: The simple principles and complex reality. Orient. Insects 1994, 28, 309-351. [CrossRef]

47. Massoud, Z.; Gruia, M. Collemboles Arthropléones de Cuba récoltés en 1969 par la mission cubano-roumanie. In Résultats des expéditions biospéologiques cubano-roumaines à Cuba, 1st ed.; Orghidan, T., Nuñez-Jiménez, A., Botosaneanu, L., Decou, V., Negrea, S., Viña-Bayés, N., Eds.; Academia Republicii Socialiste România: Bucharest, Romania, 1973; Volume 1, pp. 327-343.

(C) 2020 by the authors. Licensee MDPI, Basel, Switzerland. This article is an open access article distributed under the terms and conditions of the Creative Commons Attribution (CC BY) license (http://creativecommons.org/licenses/by/4.0/). 